\title{
A Rare Case of Bullous Pyoderma Gangrenosum in a Patient with Acute Myelogenous Leukemia
}

\author{
Veronica Marie E. Ramos, Charmaine Vanessa S. Chamberlin and Belen L. Dofitas \\ Department of Dermatology, College of Medicine and Philippine General Hospital, University of the Philippines Manila
}

\begin{abstract}
A 67-year-old female with acute myelogenous leukemia, presented with a two-week history of enlarging ecchymosis-like plaques with hemorrhagic bullae on the right forearm and anterior legs, associated with fever, pain, and swelling. Tissue cultures were persistently negative. Lesions progressed despite broad-spectrum antibiotic coverage. Histopathology showed neutrophilic dermatitis, consistent with pyoderma gangrenosum. The patient was diagnosed with the bullous type. This type is rare with only two reported cases in the Philippines since 2011. Systemic glucocorticoids were given with note of dramatic improvement of the lesions.
\end{abstract}

Key Words: bullous pyoderma gangrenosum, pyoderma gangrenosum, neutrophilic dermatoses, acute myelogenous leukemia

\section{INTRODUCTION}

Bullous pyoderma gangrenosum $(\mathrm{PG})$ is a rare and atypical presentation of the neutrophilic dermatoses. It is characterized by grouped vesicles that rapidly spread and coalesce to form large bullae and ulcerations with central necrosis and peripheral erythema. The bullous type which has a poor prognosis is usually seen in patients with lymphoproliferative hematologic disease, most commonly acute myelogenous leukemia (AML).

\section{CASE DESCRIPTION}

This is a case of a 67-year-old Filipino female, a known acute myelogenous leukemia patient for five months who already underwent the first cycle of chemotherapy. She initially presented with a two-week history of multiple, enlarging, ecchymosis-like plaques with overlying superficial hemorrhagic bullae on the right forearm and anterior lower legs. This was associated with fever, pain, swelling, and tenderness around the area with no other systemic symptoms. On physical examination, there was a welldefined, violaceous, infiltrated plaque with round, central ulceration containing hemorrhagic clots on the patient's right shin. There was surrounding erythema, edema, warmth, and tenderness (Figure 1A). A similar smaller lesion was

E-Poster presented at the $42^{\text {nd }}$ Annual Convention of the Philippine Dermatological Society, November 5-7, 2019, Edsa Shangri-La, Mandaluyong City, Philippines.

Corresponding author: Veronica Marie E. Ramos, MD

Department of Dermatology

Philippine General Hospital

University of the Philippines Manila

Taft Avenue, Manila 1000, Philippines

Email:vmeramos@gmail.com seen on the left leg as an infiltrated, violaceous plaque with overlying flaccid bulla. Superficial sloughing of the roof of the bulla was seen on the medial side (Figure 1B). There was also a well-defined, flaccid, serosanguinous, fluid-filled bulla on the right arm with an underlying erythematous, warm, and tender base (Figure 1C).

An initial biopsy was taken from the arm, showing neutrophilic dermatitis with septal panniculitis, consistent 


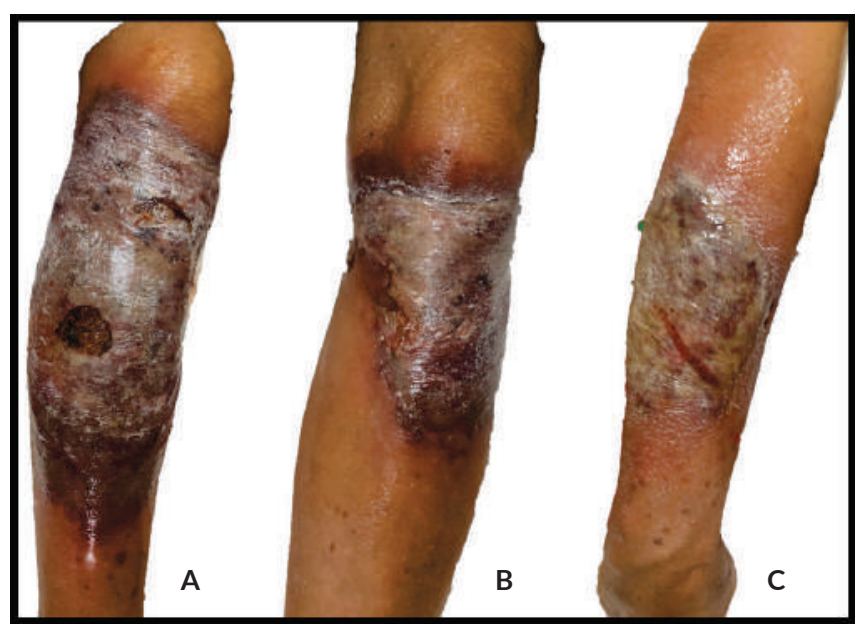

Figure 1. (A) Anterior view of the right leg showing a welldefined violaceous infiltrated plaque with round central ulceration containing hemorrhagic clots. There is surrounding erythema and edema. (B and C) Smaller violaceous plaques with overlying flaccid bullae seen on the left leg and right forearm.

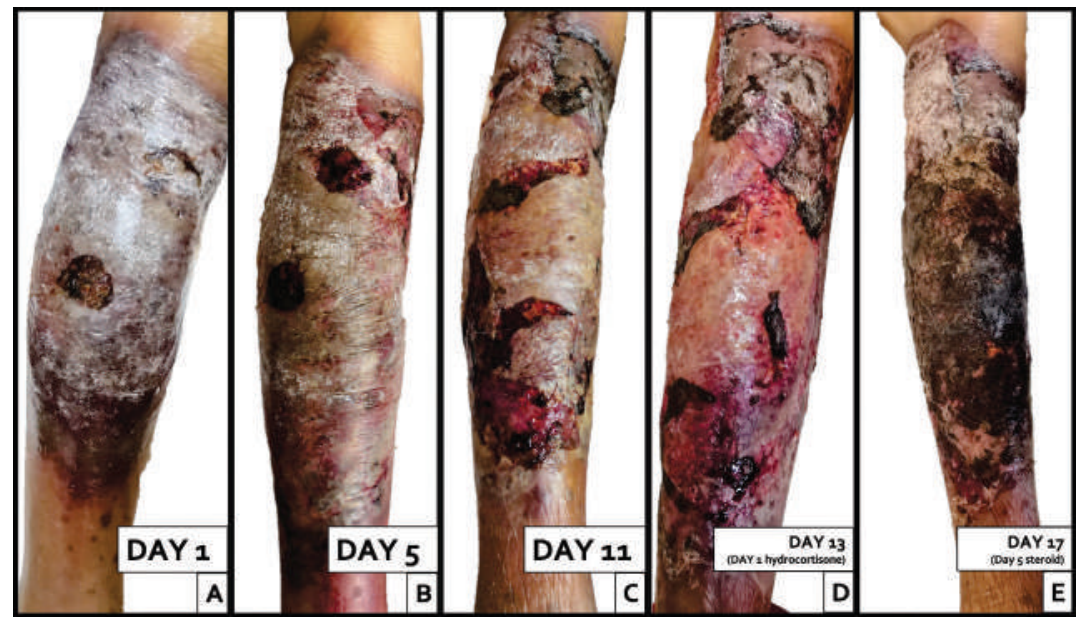

Figure 2. (A to D) Progression noted as an increase in induration, necrosis, and infiltration of the borders with blue-gray color. (E) Drying up of the lesion clinically apparent after five days of high dose corticosteroids.

with cellulitis. While awaiting work-up, the patient was maintained on broad-spectrum antibiotics, meropenem, and vancomycin. Initial tissue cultures were negative for bacterial growth. Clinically however, there was noted progression of the lesions with increase induration and infiltration of the borders with a blue-gray violaceous color (Figures 2A-D). Repeat biopsy and tissue cultures were done. Histopathology of a well-developed lesion on the leg showed neutrophilic dermatitis, now consistent with pyoderma gangrenosum (Figure 3). Repeat cultures were persistently negative. With the clinical progression of the lesions despite antibiotic treatment, and with negative bacterial cultures, the patient was diagnosed with bullous pyoderma gangrenosum. The patient was eventually started on systemic glucocorticoids, hydrocortisone $100 \mathrm{mg}$ IV q12. Lesions were dressed with petroleum jelly impregnated gauzes twice daily. Upon starting appropriate treatment, there was rapid drying up of the lesions with an associated decrease in pain and swelling (Figure 2E). The patient was discharged improved after 18 hospital days. Subsequent follow-up consultations showed progressive improvement. The high dose steroid was slowly tapered until discontinued after 16 weeks of treatment. The plaques overlying the previous ulcers were treated with hydrogel dressings to soften the thick black crusts. These eventually left hypopigmented cribriform scarring with areas of hypertrophy after 6 months (Figure 4). The patient is 

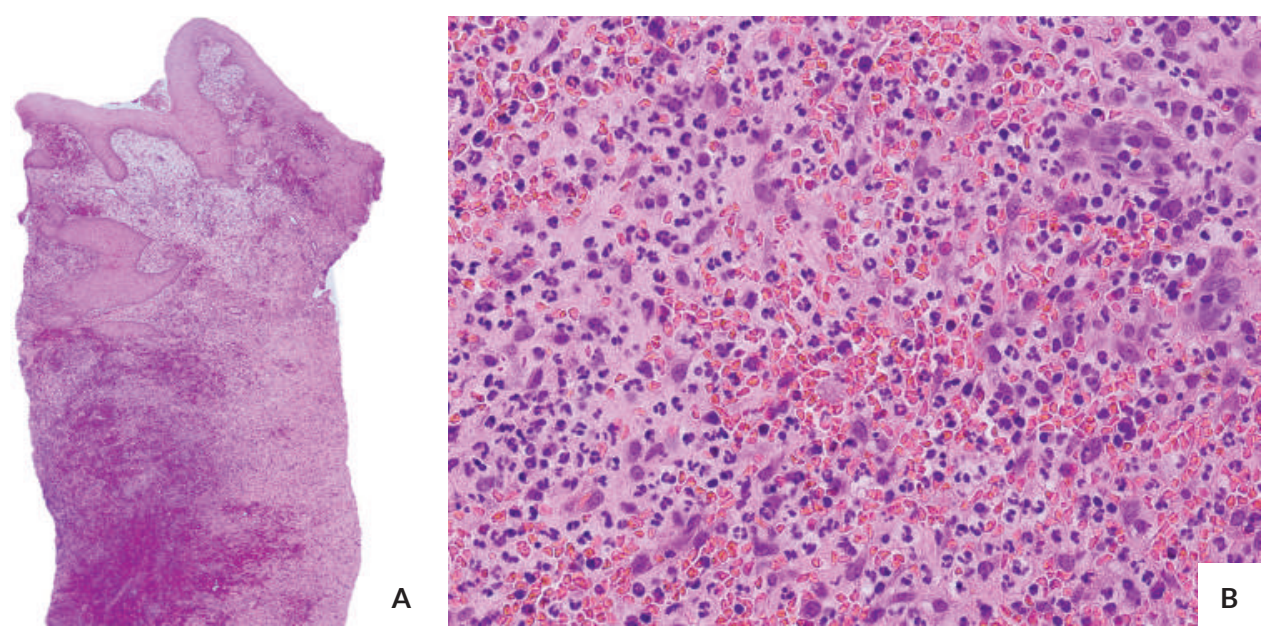

Figure 3. Histopathology of the ulcerated plaque on the leg on H\&E showing pseudocarcinomatous epidermal hyperplasia, spongiosis, with dense infiltrate of predominantly neutrophils. Signed out as neutrophilic dermatitis.
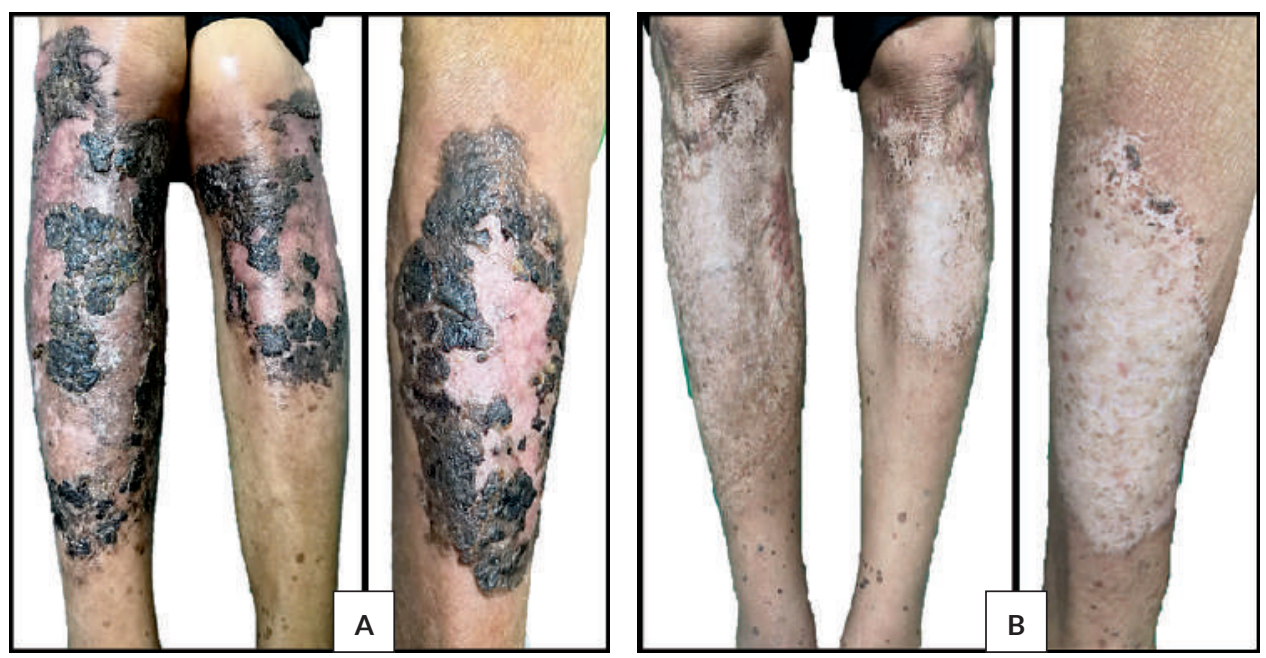

Figure 4. (A) Subsequent follow- up at six weeks after discharge showing decreased edema and inflammation with thick black crusts overlying the previously ulcerated areas of the legs and forearm. (B) Hypopigmented patches with areas of cribriform scarring at 6 months after the initial appearance of the lesions.

still on long term monitoring for any recurrence. She is on regular follow-up with her hematologist and had completed four cycles of chemotherapy. She is due for repeat bone marrow aspiration biopsy for monitoring of her leukemia.

\section{DISCUSSION}

The term pyoderma gangrenosum (PG), coined in the 1930s has long been a misnomer. During that time, it referred to purulent skin infections that had extensive necrotic features. ${ }^{1,2} \mathrm{PG}$ is now defined as a rare, chronic, non-infectious, neutrophilic, inflammatory dermatosis. ${ }^{3}$ It is uncommon with only three to ten cases per million people per year. ${ }^{4}$ Local data from the Philippine Dermatological
Society- Health Information System, reported 122 cases of PG since 2011. Only 2 of these are of the bullous type. In our institution, only five cases of PG were biopsy-proven since 2011. There were no recorded bullous types.

The bullous type of PG is one of the four general classifications of the disease, namely, the ulcerative or classic type, bullous type, pustular, and vegetative type. These vary in clinical presentation, epidemiology, and prognosis. ${ }^{3}$ Although helpful in differentiating from other disease entities, histopathologic studies are not pathognomonic. The resulting changes depend on the clinical type, the stage of the disease, and the biopsy site. Inactive, expanding lesions, typical changes include dense dermal infiltrate composed of neutrophils occasionally extending to the subcutis. ${ }^{5}$ 
The etiology of the pyoderma gangrenosum is unknown. PG represents a complex pattern of different contributing factors which include, neutrophil dysfunction, genetic mutations, and abnormal inflammation. ${ }^{6}$ It is mostly related to several distinct diseases. In a systematic review by De Filippis, reviewing 208 articles with 823 cases of PG, inflammatory bowel disease was seen in more than $60 \%$ of cases. Arthritis and hematologic disorders follow at 16 and 12 percent, respectively. ${ }^{7}$ Our patient has acute myelogenous leukemia, of which the bullous types are commonly seen.

Clinically, the bullous type is characterized by initially painful vesicles progressing to inflammatory bullae, with rapid evolution into ulcers, necrotic areas, and a peripheral halo of erythema. ${ }^{8}$ In a review of 38 bullous PG cases by Sakiyama, the mean age of appearance is 51 years with a female predominance. Twenty-five out of these 38 cases had concurrent hematologic malignancy of which AML was the most common. ${ }^{8}$ These findings are consistent with the demographic profile of our patient, a 57-year-old female with AML. Several differentials would come to mind in patients presenting with infiltrated bullous lesions, such as infections, vascular diseases, malignancies, and neutrophilic dermatosis. Considering the clinical picture of the patient, with signs of inflammation, it is prudent to rule out any underlying infectious process which may commonly present in immunocompromised patients such as in malignancies. Bullous type of cellulitis was the first consideration. The background of a hematologic malignancy, neutrophilic dermatoses, specifically the atypical bullous types were also considered. Hence, cellulitis like sweet syndrome and bullous pyoderma gangrenosum were the other differentials. With supporting laboratory examinations, histopathology, demographics, and associated diseases, bullous pyoderma gangrenosum was diagnosed in our patient.

The appearance of bullous types of pyoderma in AML patients signifies poor prognosis. Hematologic disorders with associated PG tend to be more aggressive with $75 \%$ of patients dying within one year without treatment., Treatment of the underlying condition along with appropriate systemic therapies is important. As with all types of pyoderma gangrenosum, treatment depends on the type, severity, and rapidity of disease. First-line recommended therapy includes medium to high dose corticosteroids at 0.5 to $1 \mathrm{mg} / \mathrm{kg} /$ day. ${ }^{10} \mathrm{~A}$ high initial response rate is expected with systemic steroids. Our patient received an equivalent of $1 \mathrm{mg} /$ $\mathrm{kg} /$ day of hydrocortisone intravenously, gradually tapering and shifting to oral prednisone as the disease stopped to progress and signs of improvement were seen. Local wound management with moisture-retentive dressings was applied, avoiding any debridement that may cause pathergy. ${ }^{10}$

\section{CONCLUSION}

In patients with hematologic malignancies, a diagnosis of a neutrophilic dermatosis, especially the atypical bullous type, should always be a differential diagnosis. Bullous pyoderma gangrenosum is rare with only two recorded cases in the Philippines since 2011. Other considerations such as an infectious process should be properly ruled out. The early recognition of bullous pyoderma gangrenosum is of utmost importance because the timely administration of treatment with glucocorticoids or other immunosuppressive agents can lead to a rapid resolution.

\section{Statement of Authorship}

All authors participated in data collection and analysis, and approved the final version submitted.

\section{Author Disclosure}

All authors declared no conflicts of interest.

\section{Funding Source}

This paper was funded by the authors.

\section{REFERENCES}

1. Brunsting LA. Pyoderma (Echthyma) Gangrenosum. Arch Dermatol. 1982; 118(10):743-68.

2. Farhi D. The clinical and histopathological description of geometric phagedenism (Pyoderma Gangrenosum) by Louis Brocq one century ago. Arch Dermatol. 2008; 144(6):755.

3. Sewon K. Fitzpatrick's Dermatology in General Medicine. New York: Mcgraw-Hill Education; 2018.

4. Ruocco E, Sangiuliano S, Gravina AG, Miranda A, Nicoletti G. Pyoderma gangrenosum: an updated review. J Eur Acad Dermatol Venereol. 2009; 23(9):1008-17.

5. Gameiro A, Pereira N, Cardoso JC, Gonçalo M. Pyoderma gangrenosum: challenges and solutions. Clin Cosmet Investig Dermatol. 2015; 8:285-93.

6. Alavi A, French LE, Davis MD, Brassard A, Kirsner RS. Pyoderma Gangrenosum: an update on pathophysiology, diagnosis and treatment. Am J Clin Dermatol. 2017; 18(3):355-72.

7. DeFilippis EM, Feldman SR, Huang WW. The genetics of pyoderma gangrenosum and implications for treatment: a systematic review. Br J Dermatol. 2015; 172(6):1487-97.

8. Sakiyama M, Kobayashi T, Nagata Y, Fujimoto N, Satoh T, Tajima S. Bullous pyoderma gangrenosum: a case report and review of the published work. J Dermatol. 2012; 39(12):1010-5.

9. Sener S, Sarac G, Altunisik N, Bayindir Y. Delayed diagnosis of bullous pyoderma gangrenosum with acute myelogenous leukemia. Niger J Clin Pract. 2016; 19(6):837-9.

10. Miller J, Yentzer BA, Clark A, Jorizzo JL, Feldman SR. Pyoderma gangrenosum: A review and update on new therapies. J Am Acad Dermatol. 2010; 62(4):646-54. 